\title{
Transgenerational effects of ancestral prenetal stress on the gut-brain axis
}

\author{
Crystal Mulik ${ }^{1}$, Hannah Scott ${ }^{1}$, Douglas Inglis ${ }^{2}$, Tony Montina ${ }^{3}$, Gerlinde A.S. Metz ${ }^{1}$
}

${ }^{1}$ Canadian Centre for Behavioral Neuroscience, University of Lethbridge, 4401 University Drive, Lethbridge, Alberta, Canada T1K 3M4

${ }^{2}$ Agriculture and Agri-food Canada, 5403 1st Ave South, Lethbridge, Alberta, Canada

${ }^{3}$ Department of Chemistry and Biochemistry, University of Lethbridge, 4401 University Drive, Lethbridge, Alberta, Canada T1K 3M4

\section{Abstract}

The effects of ancestral prenatal stress can propagate across generations to alter the wellbeing of directly and indirectly exposed descendants via epigenetic mechanisms. Prenatal stress has been shown to alter the function of the gut-brain axis, a bi-directional signaling pathway between the gut microbiome and the enteric and central nervous systems. There has been no study investigating the impact of remote prenatal stress in ancestors on the gutmicrobiome connection. Here we investigated if exposure to transgenerational ancestral stress affects the gut-brain axis through changes in the microbiome and microbiota. A multigenerational rat cohort consisting of a F0, F1, F2, and F3 generation was utilized in this study. Pregnant dams in the F0 generation were exposed to repeated restraint stress and overnight social isolation from gestational days 12-18. Breeding of three successive generations occurred in the absence of gestational stress along with a lineage of yoked controls. Fecal collection occurred for males and female in each generation at the age of 30 days, 90 days, and 115 days. Fecal samples were analyzed using $1 \mathrm{H}-\mathrm{NMR}$ spectroscopy to examine the metabolome. The data are being analysed using supervised and unsupervised machine learning approaches. The data are expected to reveal that the fecal metabolome is characteristically altered by ancestral prenatal stress in each generation, resulting in a biomarker signature that is linked to the behavioural phenotype. We predict changes in the gut metabolome and microbiome to be most significant in the F3 generation. These findings could lead to further understanding of intestinal dysbiosis and its impact on the brain, and sex-specific metabolic biomarkers that are predictive of stress-associated adverse health outcomes.

Cite as: Mulik C., Scott, H., Inglis, D., Montina, T., Metz, G.A.S. 2019. Transgenerational effects of ancestral prenetal stress on the gut-brain axis. Alberta Academic Review, Vol 2 (3) 18, CASCH Special Issue (not peer-reviewed), DOI: 10.29173/aar109. 